\title{
APLICABILIDADE DA TEORIA DOS JOGOS AO INSTITUTO DA MEDIAÇÃO
}

\author{
Camila Stabach Mendes ${ }^{1}$
}

\section{RESUMO}

O estudo objetiva analisar a aplicabilidade da Teoria dos Jogos ao Instituto da Mediação. Utilizou-se técnica de metodologia dedutiva e dialética, partindo do método teóricobibliográfico. São elencados os métodos heterocompositivos e autocompositivos. Faz-se análise do instituto da mediação, analisando conceituação, principais escolas e princípios. Aborda-se a Teoria dos Jogos, destacando os pressupostos axiomáticos, bem como contribuição de John Nash. Observa-se a possibilidade de aplicação de conceitos da Teoria dos Jogos ao instituto da mediação. Conclui-se que a opção pela cooperação é economicamente eficiente, gerando solução com maximização dos interesses individuais para os envolvidos.

Palavras-chave: Autocomposição; Métodos alternativos de resolução de conflitos; Mediação; Teoria dos Jogos; Equilíbrio de Nash

\section{APPLICABILITY OF GAME THEORY TO MEDIATION INSTITUTE}

\begin{abstract}
The study aims to analyze the applicability of Game Theory to mediation institute. Deductive and dialectical methodology technique was used, starting from theoretical-bibliographic method. The heterocomposite and autocomposite methods are listed. An analysis of the mediation institute is carried out, analyzing its conceptualization, main schools and principles. Game Theory is approached, highlighting the axiomatic assumptions, as well as the contribution of John Nash. The possibility of applying concepts from Game Theory to the institute of mediation is observed. It is concluded that the option for cooperation is economically efficient, generating a solution that maximizes individual interests for those involved.
\end{abstract}

Keywords: Self-composition; Alternative methods of conflict resolution; Mediation; Game Theory; Nash's balance

\footnotetext{
1 Mestranda em Direito Empresarial e Cidadania pelo pelo Centro Universitário Curitiba UNICURITIBA, Brasil; Curso de Extensão Universitária em Comércio Internacional: desafios sociais, jurídicos e económicos num mundo globalizado pela Faculdade de Direito da Universidade de Coimbra, UC, Portugal (2017); Formação complementar em Direito e Empresa na Prática: Desafios Contemporâneo, pelo Centro Universitário Curitiba, UNICURITIBA, Brasil (2018); Formação complementar em Direito Empresarial Aplicado: Contabilidade e Relações Negociais, pelo Centro Universitário Curitiba, UNICURITIBA, Brasil (2019); Advogada; Mediadora Privada; E-mail: camila.s.mendes@icloud.com
} 


\section{INTRODUÇÃ̃}

A sociedade está em constante transformação, algumas mudanças acabam por influenciar de maneira mais marcante a vida do ser humano. Os avanços tecnológicos, mais precisamente o desenvolvimento e o acesso a internet, são uma dessas mudanças que impactaram diretamente o modo como o homem se relaciona, consome e trabalha.

A internet possibilitou a expansão das relações humanas, de modo antes inimaginável, não se verifica limitações que outrora eram comuns, como por exemplo a limitação territorial, não se restringe mais o consumo e o trabalho a região onde a pessoa habita ou empresa está constituída. Atualmente pessoas e empresas conseguem se relacionar com os demais independentemente de onde se encontram, o que permitiu a conexão de indivíduos de diferentes culturas, realidades, costumes.

Fato que além de proporcionar maior desenvolvimento também ocasionou um maior conflito de interesses, o que resulta no aumento da conflituosidade. O conflito é uma fato inevitável e inerente ao comportamento humano. Uma sociedade sem conflito, seria utópica e estática. O conflito quando bem abordado possui efeitos positivos, pode-se verificar como um propulsor da evolução, pois é a partir do conflito que as relações são aprimoradas e amadurecidas.

Compreendida a importância do conflito, o que deve se buscar não é sua extinção, mas sim a melhor maneira de abordá-lo. A objetivação da extinção do conflito pode ser uma maneira utópica de análise da problemática. Todavia, dado seu crescimento em razão das alterações sociais observadas, é importante buscar ferramentas para que se verifique uma reação saudável frente aos impasse, possibilitando que a sociedade seja então impacta com os seus efeitos positivos de evolução e amadurecimento de relações. Buscando somente evitar pontos negativos do conflito como o rompimento de laços, que se verifica quando os envolvidos não abordam de maneira correta o impasse entre interesses.

Há diversos modelos e formas para se abordar o conflito, de modo que para cada situação pode se elencar um modelo como o método mais adequado para a solução. Desse modo, coloca-se a seguinte problemática: há viabilidade da aplicação da Teoria dos Jogos à eventual aprimoramento e sofisticação do instituto da Mediação?

Para enfrentamento da problemática apresentada, optou-se pela utilização da técnica de metodologia dedutiva e dialética, partindo do método teórico-bibliográfico, com apoio em pesquisa doutrinária. 
No presente estudo, em primeiro momento, serão elencados os métodos heterocompositivos e autocompositivos de resolução de conflitos, sendo que haverá escrutínio produzido com maior cautela a respeito das modalidades autocompositivas, diferenciados os modelos existentes por meio de abordagem de seus principais aspectos. Em sequência será feita análise a respeito do instituto da mediação, como método de resolução de conflitos, analisando sua conceituação e abordando suas principais escolas e princípios.

Ato contínuo, será traçada uma abordagem a respeito da da Teoria dos Jogos, destacando-se os pressupostos axiomáticos sobre os quais se funda a teoria. Busca-se traçar abordagem verticalizada sobre as contribuição de John Nash à Teoria dos Jogos, haja vista a teoria criada pelo referido autor, a qual se denomina Teoria do Equilíbrio de Nash, verificarse-á como a teoria foi responsável por expandir o escopo da Teoria do Jogos, uma vez que, a noção de equilíbrio, defendida pelo autor, não se restringia apenas aos jogos de soma zero.

Por fim, será observada a possibilidade de aplicação de conceitos da Teoria dos Jogos ao instituto da mediação. Verificar-se-á quais são os pontos de convergência da teoria com o instituto e possíveis benefícios desta analise conjunta.

\section{A MEDIAÇÃo ENQUANTO METODO AUTOCOMPOSITIVO DE RESOLUÇÃo DE CONFLITOS}

Os método de resolução de conflitos podem ser classificado em autocompositivo ou heterocompositivo. Quanto a característica heterocompositiva, diz-se que "o conflito é administrado por um terceiro, escolhido ou não pelos litigantes, que detém o poder de decidir, sendo a referida decisão vinculativa em relação às partes." (SANTOS, 2004, p. 14 apud KESSLER; TRINDADE, 2019). Diante dessa perspectiva, pode-se elencar como exemplos desta modalidade a arbitragem e as vias judiciais.

Já no tocante aos métodos autocompositivos, estes decorrem do fato que "as próprias partes interessadas, com ou sem a colaboração de um terceiro, encontram, através de um consenso, uma maneira de resolver o problema." (SANTOS, 2004, p. 14 apud KESSLER; TRINDADE, 2019). Das formas autcompositivas encontramos a negociação, a conciliação e a mediação.

Haja vista o objetivo do trabalho, passa-se analisar com maior detalhamento, um dos métodos autocompositivos, mais especificamente a mediação. Porém para boa compreensão 
deste instituto faz-se necessária a distinção da mediação para com os outros métodos autocompositivos.

A mediação e a conciliação possuem algumas semelhanças, como pontua Tartuce (2021):

São pontos comuns à mediação e à conciliação: 1 . A participação de um terceiro imparcial; 2. A promoção da comunicação entre os envolvidos; 3. A não imposição de resultados; 4 . $\mathrm{O}$ estímulo à busca de saídas pelos envolvidos; 5 . O exercício da autonomia privada na elaboração de opções para os impasses.

Há que se salientar que parte da doutrina sustenta não haver diferença entre mediação e conciliação. Porém como sustentado por Lília Maria de Morais Sales ( apud TARTUCE, 2021) "Na conciliação o conciliador sugere, interfere, aconselha. Na mediação, o mediador facilita a comunicação, sem induzir as partes ao acordo". Ademais, destaca-se a diferença na profundidade da abordagem realizada, sendo a abordagem feita dentro da mediação mais extensa.

No que tange à negociação, esta se difere da mediação por não haver um terceiro facilitador do dialogo. Na negociação entende-se que o negociador é a própria parte interessada ou alguém que lhe represente que deseja chegar a um acordo que satisfaça seus interesses substantivos. (URY; FISHER,).

Entendida a diferenciação da mediação para os demais métodos autocompositivos, faz-se mister a realização de análise verticalizada a respeito da conceituação do instituto da mediação, analisando suas escolas e principais características que vão ao encontro das ideias que sedimentaram a mediação, mas que apresentam traços distintos quando analisados sob a perspectiva de sua operacionalização.

A mediação consiste em um meio consensual de abordagem de controvérsias, no qual um terceiro imparcial atua para facilitar a comunicação entre os envolvidos e propiciar que eles possam, a partir da percepção ampliada dos meandros da situação controvertida, protagonizar saídas produtivas para os impasses que os envolvem. (TARTUCE, 2021)

Há vários modelos ou escolas de medição, as quais utilizam-se de diferentes métodos de execução do instituto, sendo o Modelo de Harvard (tradicional linear), o Modelo Transformativo (Bush e Foger) e o Modelo Circular Narrativo (Sara Cobb) os mais utilizados nos países que praticam a mediação de conflitos (KESSLER; TRINDADE, 2019). 
A escola de mediação linear ou mediação tradicional, foi concebida a partir do projeto de negociação de Harvard, se desenvolveu como alternativa à barganha posicional, modelo surgiu dentro da mediação empresarial, sendo que sua principal ferramenta é a negociação, possui como principais autores Roger Fisher e Willian Ury.

Segundo Roberto Portugal Bacellar, a referida escola é um desdobramento da negociação baseada em princípios, tendo um processo estruturado linearmente em cinco fases bem definidas, com o intuito de restabelecer a comunicação entre as partes para identificar os interesses encobertos pelas posições e, com isso, alcançar um acordo. (BACELLAR, 2012).

A Escola de Harvard, é considerada a mais importante no estudo da negociação, uma vez que esta se propõem a trabalhar os possíveis caminhos para vencer cada dia um dos obstáculos, introduzindo conceitos claros e essenciais que são incorporados à mediação. Entre eles, a diferença entre posições e interesses fato que mudou a visão da vontade das partes, de manifesta à subjacente, mostrando a importância da escuta e da investigação. (OLIVEIRA, 1999. p. 119).

Nesse modelo é adotado uma linha de mediação passiva, onde não ocorre uma intervenção direta do mediador, mas sim, uma posição menos invasiva, de facilitador do diálogo, utilizando-se de técnicas para alcançar o objetivo principal da mediação TradicionalLinear de Harvard, que é a construção do acordo.

Essa escola encontra críticas em razão de não ter como escopo trabalhar os sentimentos das partes e suas relações, mas basicamente encontrar um acordo mutuamente vantajoso (KESSLER; TRINDADE, 2019).

A Escola Circular-Narrativa, como o seu método desenvolvido, principalmente pela professora norte americana Sara Cobb, agrega ao modelo de Harvard, premissas da teoria dos sistemas de Niklas Luhmann.

O modelo fundamenta-se na comunicação circular, que consiste na desconstrução das narrativas iniciais através de perguntas que estimulam a mudança de foco do problema, trazendo uma nova perspectiva a questão vivenciada, visando fomentar a reflexão sobre os pontos apresentados. (KESSLER; TRINDADE, 2019).

Nessa espécie de mediação, o foco se centraliza na necessidade de compreensão da outra parte, ou seja, usar da empatia para entender as particularidades, interesses, objetivos e características da outra pessoa. Pode-se se dizer que, diferente do modelo linear de Harvard, através da escola circular-narrativa não se analisa a causa imediata que originou aquele problema - a causalidade não é mais imediata -, mas o 
conjunto de causas remotas, anteriores, que, de alguma forma, contribuíram para que o conflito se desenvolvesse. (KESSLER; TRINDADE, 2019)

O modelo tem como objetivo a mudança de perspectiva, de modo que ao fim das sessões de mediação, os mediados passem a visualizar o fato passado com outro enfoque, trazendo novas perspectivas, o que proporciona uma visão prospectiva de colaboração, solidariedade e empatia.

O Modelo Transformativo, criado por Robert A. Barush Bush (teórico da Negociação) e Joseph F. Folger (teórico na comunicação) tem como objetivo situar o acordo como uma possibilidade, diferentemente do modelo de Harvard que coloca este como principal objetivo.

Possui como base os novos modelos comunicacionais e seu objeto central verifica-se na relação interpessoal dos mediados, de modo que o mediador deve incentivar a participação destes, bem como trabalhar para que as partes ressignifiquem os pontos controvertidos e negativos, de maneira positiva e construtiva. $\mathrm{O}$ modelo não se limita apenas à obtenção de um acordo, mas o sim reaproximar as partes, abordar seu aspecto emocional, afetivo, financeiro, etc (FERIOLI, 2012).

Os doutrinadores Armando Sérgio de Toledo, Jorge Tosta e José Carlos Ferreira Alves (2014, p. 183, apud KESSLER; TRINDADE, 2019), ao tratar dessa escola, assim esclarecem:

\footnotetext{
Os adeptos dessa corrente querem se distanciar da tradição de mera "solução de problemas" na mediação, buscando mudar o paradigma da visão de mundo individual e relacional; para essa corrente, as disputas não devem ser vistas como problemas, mas sim como oportunidades de crescimento moral e transfor-mação. Nessa concepção, empoderamento e reconhecimento são os dois mais relevantes efeitos que a mediação pode gerar e atingi-los é o objetivo mais importante. (DE TOLEDO; TOSTA; ALVES, 2014 apud KESSLER; TRINDADE, 2019).
}

Destaca-se que os atuantes desta escola trabalham com o conflito em sua integralidade, abordando tanto o aspecto emocional, afetivo, financeiro, legal, entre outros que possam ser encontrados na situação fatiga apresentada. Trabalha para que as partes visualizem um aos outros como protagonistas, e como responsáveis pela transformação da situação.

Faz-se necessário ao investigar o instituto, também mencionar seus princípios. Primeiramente, tem-se a Autonomia da Vontade e Decisão Informada, princípio que está 
intimamente ligado a voluntariedade, de modo que a mediação só poderá ser realizada com a concordância de todos os envolvido, além disso, este princípio pode ser associado à ideia de protagonismo do mediado na tomada de decisão (TARTUCE, 2021).

Outro princípio a ser listado é o da Informalidade e Independência. Não há um procedimento ou forma pré estabelecidos, de modo que a mediação pode ser construída da forma que se julgar mais eficiente para o restabelecimento da comunicação, sendo que o mediador não está subordinada ou influenciado de qualquer modo (CONSELHO NACIONAL DE JUSTIÇA,2015) .

Ademais, o Princípio da Oralidade pontua que, por se tratar de um procedimento que busca o restabelecimento da comunicação, a mediação se pauta no dialogo e conversação. Além disso, o princípio da imparcialidade pode ser traduzido na equidistância do mediador em relação aos envolvidos no conflito, é um elemento crucial para que seja reconhecida como válida a atuação do terceiro que interventor . (CONSELHO NACIONAL DE JUSTIÇA,2015)

Há que se destacar o Princípio da Busca do Consenso, Cooperação e Não Competitividade. A mediação busca a cooperação das partes, superando a visão adversária do conflito, objetivando construir uma solução de benefício para ambas as partes envolvidas. Já o princípio da Boa-fé preconiza que a mediação é um meio consensual que envolve a participação voluntária dos participantes na conversa, sendo essencial que haja disposição e boa-fé para que possam se comunicar . (TARTUCE, 2021).

O Princípio da Confidencialidade estabelece que a mediação é confidencial, e necessita que assim seja para que as partes consigam se sentir seguras para se expressar livremente durante a sessão. Por fim o princípio da isonomia determina que a mediação sempre busca conceder tratamento igualitário entre as pastes, tratando todos do mesmo modo e buscando conceder a todos as mesmas oportunidades . (TARTUCE, 2021).

Compreendidas as principais características do instituto da mediação, passa-se a analise a Teoria dos Jogos, sendo necessário traçar abordagem que diga respeito a sua constituição, bem como seus pressupostos axiomáticos e seus aspectos que vão ao encontro da teoria fundada por John Nash, o qual é criador da notadamente difundida ideia de cooperação tratada pela "Teoria do Equilíbrio de Nash".

\section{A TEORIA DOS JOGOS E SEUS PRESSUPOSTOS AXIOMÁTICOS}


A Teoria dos Jogos é um ramo da matemática aplicada, que através de uma visão interdisciplinar do estudo do comportamento humano e fornece as bases para que se entenda a interação entre os agentes envolvidos em um contexto de disputa. (VITALE; SILVA, 2017)

Para os autores Brígida Alexandre Santini, Gilmar Garbugio, Humberto José Bortolossi, Polyana Alves Santos e Larissa Santana Barreto (2010, apud NETO; MENDONÇA 2013) a Teoria dos Jogos pode ser definida da seguinte forma:

\begin{abstract}
A teoria dos jogos é uma teoria matemática criada para se modelar fenômenos que podem ser observados quando dois ou mais agentes de decisão interagem entre si. Ela fornece a linguagem para a descrição de processos de decisão consistentes objetivos envolvendo mais do que um indivíduo.
\end{abstract}

Por sua vez, de acordo com Alex Allochio Scalfoni (2013):

\begin{abstract}
“a Teoria dos Jogos pode ser definida basicamente como uma ferramenta matemática que visa a analisar uma situação de conflitos entre agentes racionais, com o objetivo de descobrir as melhores estratégias a serem utilizadas para que tais participantes alcancem o objetivo que desejam."
\end{abstract}

Segundo Almeida (2003) a teoria dos jogos é: "em linhas gerais, a análise matemática de qualquer situação que envolva um conflito de interesses, com o fito de descobrir as melhores opções(...)"

O objeto de estudo desta teoria é o conflito, dentro de uma situação de conflito os envolvidos precisam desenvolver estratégias para maximizar seus ganhos, a partir de certas regras preestabelecidas. (BORBA; FIBRANS; COSTA, 2016)

Faz-se necessário ressaltar que cada conflito, ou situação de interação estratégica, possui suas próprias características sendo necessário modelos diversos para aborda-lá. Neste sentido:

\footnotetext{
Não é possível tratar de todas as situações de interação estratégica com o mesmo modelo, uma vez que há diferentes tipos de situações de interação: há interações que acontecem apenas uma vez e nas quais os agentes envolvidos decidem simultaneamente; outras que se repetem no tempo; outras em que os agentes envolvidos decidem em uma ordem bem-definida; outras em que alguns decidem já conhecendo as decisões dos outros agentes etc.(FIANI, 2015)
}

Sendo assim a análise de qualquer jogo ou situação de conflito deve ser iniciado com a escolha de um modelo que melhor se adeque a situação (FIANI, 2015). 
Para melhor compreender esses modelo faz-se necessário destacar os pressupostos axiomáticos sobre os quais se funda a teoria.

Primeiro pressuposto analisado é o dos agentes ou jogadores. Segundo Ronaldo Fiani (2015, p13) : "Um jogador é qualquer indivíduo ou organização envolvido no processo de interação estratégica que tenha autonomia para tomar decisões".

Ademais, outro pressuposto analisado é a ideia de utilidade. Este conceito reflete o objetivo de cada jogador de garantir a maior satisfação possível com o jogo (ALMEIDA, 2003). Segundo, Alex Allochio Scalfoni (2013): "enquanto axioma da Teoria dos Jogos, a noção de utilidade é uma reflexão do objetivo do jogador, que é o de garantir a maior satisfação possível, dadas as circunstâncias, com o jogo".

Importante destacar a este axioma não possuo um valor absoluto, nesse sentido exemplifica Almeida (2003):

\footnotetext{
Não é um valor absoluto: a utilidade só tem valor relativamente à utilidade de outro resultado. Por exemplo: se se sabe que uma pessoa prefere viajar para passar as férias na praia a andar de cavalo, para ela, a utilidade de viajar para a praia é maior do que a de andar a cavalo. Neste caso, um jogador racional seria o que preferisse a estratégia que o permitisse viajar para a praia. Normalmente, diz-se que o jogador racional é aquele que pretende sempre maximizar seus ganhos médios. Contudo, nem sempre tal ocorre, porque os jogadores podem ter objetivos diferentes. Dificilmente um jogador arriscaria ganhar R $\$ 1.000 .000,00$ incertos se tivesse que abrir mão de R\$ 100.000,00 já certos. Neste caso, a utilidade de R\$100.000,00 é maior que a de $\mathrm{R} \$ 1.000 .000,00$.
}

Outro axioma a ser analisado é o de presunção de racionalidade, a ideia de racionalidade presente na Teoria dos Jogos é relativamente simples, de acordo com a teoria de Neumann, a racionalidade está no individuo que busca obter o máximo de utilidade.

Segundo Almeida (2003): "a hipótese de racionalidade dos jogadores serve ao propósito de tornar mais restrita a totalidade de resultados possíveis em um jogo, já que o comportamento estritamente racional é mais previsível que o comportamento irracional".

Por fim, as estratégia e payoffs, que são o conjunto de opções de ação que os jogadores possuem para chegarem a todos os resultados possíveis . (SCALFONI, 2013).

Apresentado alguns dos axiomas da Teoria dos Jogos, bem como sua conceituação, faz-se mister trazer à tona uma análise, em linhas gerais, da Teoria do Equilíbrio de Nash e suas principais perspectivas. 
É importante salientar as contribuição de John Nash, que foram fundamentais para o desenvolvimento da teoria dos jogos. O autor além de quebrar paradigmas econômicos criados por Adam Smith, foi responsável pela criação da “ Teoria do Equilíbrio de Nash" que propõe a possibilidade de ações colaborativas entre os agentes em jogos não-cooperativos. A criação de John Nash, foi responsável por expandir o escopo da Teoria do Jogos, uma vez que, a noção de equilíbrio, defendida pelo autor, não se restringia apenas aos jogos de soma zero.

Para Azevedo (2015) O princípio do equilíbrio pode ser exposto da seguinte forma:

"a combinação de estratégias que os jogadores preferencialmente devem escolher é
aquela na qual nenhum jogador faria melhor escolhendo uma alternativa diferente
dada a estratégia que o outro escolhe. A estratégia de cada jogador deve ser a melhor
resposta às estratégias dos outros" (AZEVEDO, 2015 apud BORBA; FIBRANS;
COSTA, 2016 )

Para Fiani (2015) pode-se dizer que uma combinação de estratégias constitui um equilíbrio de Nash, "quando cada estratégia é a melhor resposta possível às estratégias dos demais jogadores, e isso é verdade para todos os jogadores".

Para Nash a cooperação não é incompatível com o pensamento de ganho individual, de modo que é possível maximizar seus ganhos cooperando com os outros participantes (ou adversário). (BORBA; FIBRANS; COSTA, 2016).

Verifica-se no artigo a Teoria dos Jogos de Henry Paulo Dias (2004), uma sintetização do conceito de equilíbrio de Nash, nesse sentido sintetiza o autor:

\begin{abstract}
O conceito de equilíbrio ou solução de (John) Nash: um dos ganhadores do Nobel de Economia, teorizou na década de 1950, chegando a uma combinação de estratégias também conhecida com a estratégia do não-arrependimento. Esta combinação de estratégias escolhidas leva a um resultado no qual nenhum dos jogadores individualmente se arrepende, ou seja, ambos jogadores não poderiam melhorar a sua situação unilateralmente, modificando a estratégia escolhida. $\mathrm{Na}$ Teoria dos Jogos, a utilização do conceito de Nash indica que cada um dos jogadores escolheu a melhor estratégia, independente da escolha do outro, isto é, cada um deles acha que não poderia ter agido de maneira melhor.
\end{abstract}

Compreendido a Teoria dos Jogos, bem como já elucidado a cerca do instituto da mediação, passa a analisar a possibilidade da aplicação da Teoria dos Jogos ao Instituto da mediação, bem como verificar se analise conjunta apresenta algum beneficio. 


\section{APLICABILIDADE DA TEORIA DOS JOGOS AO INSTITUTO DA MEDIAÇÃO E A SINERGIA DOS MEDIADOS À CONSTRUÇÃO DE ACORDOS}

Tendo em vista o fato de que o objeto de estudo da Teoria dos Jogos se encontra no conflito, verifica-se que a incorporação dos conceitos abrangidos pela referida teoria é de grande valia ao instituto da mediação. Isso se deve ao fato de a mediação trabalhar com o conflito, buscando transformá-lo ou tendo por objetivo a realização ou construção de um acordo para solucionar a questão controvertida instaurada entre as partes que inicialmente compuseram o litígio.

A teoria dos jogos, pode ser vista como uma ferramenta para auxiliar a entender teoricamente o processo de decisão de agentes que interagem entre si, a partir da compreensão da lógica da situação em que estão envolvidos (FIANI, 2015), se mostra proveitosa quando aplicada a mediação, visto que esta teoria auxilia o desenvolvimento da capacidade de raciocinar estrategicamente, fazendo com que os agentes explorem possibilidades de interação (FIANI, 2015), sendo estes um dos objetivos da mediação.

É possível identificar semelhanças entres conceitos das teorias dos jogos com determinadas ferramentas e princípios da mediação. Por exemplo, através da Teoria dos Jogos é possível alcançar à estratégia ótima para cada jogador, no qual os envolvidos de forma cooperativa alcançarão o melhor resultado para o problema (KESSLER; TRINDADE, 2019). Verifica-se a convergência da teoria ao instituto da mediação, pois a prática descrita acima se relaciona com princípios da mediação, mais precisamente com o Princípio da Cooperação.

Se não for verificada essa cooperação, os mediados se prejudicariam, pois as decisões e atitudes tomadas por um influenciam direta ou indiretamente o outro. Visto que segundo a Teoria do Equilíbrio de Nash, para que o resulta seja positivo para ambos, é preciso considerar a sua jogada, bem como a do outro mediando (DÓRIA, 2019).

Segundo Vitale e Silva (2017) verifica-se que o Equilíbrio de Nash é obtido quando: "os comportamentos dos envolvidos numa situação de disputa se estabilizam em resultados, em virtude dos quais não há incentivo para se arrepender ao analisar posteriormente a situação considerando a conduta da outra parte." Deste modo tem-se que quando verificado o Equilíbrio de Nash, estamos diante de um resultado que é denominado na mediação de WinWin, ou ganha ganha, onde verifica-se um desempenho em que apesar dos mediando realizarem concessões eles obtém resultado em que ambos verificam ganhos. 
Outro ponto que convergem a mediação e a Teoria dos Jogos, é o fato do resultado que a situação conflituosa não depende exclusivamente da atuação de um dos jogadores, ou no caso da mediação de um mediando, mas é resultado da interação das estratégias e táticas utilizadas no decorrer da sessão de mediação. Sendo que o comportamento dos envolvidos é fator de forte influência no resultado a ser buscado, e dependendo deles para se obter resultado favorável (VITALE; SILVA, 2017).

Importante ressaltar que, mesmo nos casos em que, aplicando-se a teoria dos jogos, a mediação não coloque um fim no litígio, esta se mostra eficiente, visto que auxilia na retomada da comunicação, reduzindo a assimetria informacional, e assim, reduzindo a contenda.

Ademais, a teoria econômica pressupõe que cada agente econômico maximize a utilidade de bens para extrair maior benefícios, verifica-se que, com a aplicação da teoria dos jogos ao instituto da mediação, obtém-se esta maximização de resultados buscada na teoria econômica. Assim, é possível afirmar que o modelo se mostra economicamente eficiente, dado seu baixo custo frente a eficiência obtida.

Por fim, conclui-se que a a opção pela cooperação é mais racional e economicamente eficiente, em detrimento de meios competitivos de resolução de disputas, visto que possui como consequência a otimização de resultados, gerando uma solução mais eficiente com maximização dos interesses individuais para todos os envolvidos, os quais, se agissem de forma não cooperativa, acabariam obtendo resultados menos eficientes. (KESSLER; TRINDADE, 2019).

\section{CONSIDERAÇÕES FINAIS}

Após percorrer o caminho inicialmente estabelecido para o presente estudo, cumpre destacar os pontos principais analisados, no decurso do trabalho, a respeito da aplicabilidade de conceitos da Teoria dos Jogos ao instituto da mediação.

Verificou-se como houve um aumento na conflituosidade, bem como salientou-se a importância do conflito para o desenvolvimento da sociedade, de modo que se constatou a importância para buscar meios para melhor abordar os conflitos existentes.

Para tanto foi estudado o modelo heterocompositivo e autocompositivo, como maior destaque a este, diferenciando em especial a negociação, mediação e conciliação. Ademais foi 
estudado com maior profundidade a mediação, apresentado seu conceito, escrutinou-se os seus princípios e as principais técnicas adotadas, abordando as três principais escolas, quais sejam, a Escola de Harvard, a Transformativa, e a Circular Narrativa.

Compreendido a respeito do instituto da mediação, passou-se à analise da Teoria dos Jogos, a qual, em linhas gerais, pôde ser definida como a análise matemática de qualquer situação que envolva um conflito de interesses, com o fito de descobrir as melhores opções que possibilitem uma maximização dos interesses individuais.

Foi abordado a respeito das contribuições de John Nash, as quais foram fundamentais para o desenvolvimento da teoria dos jogos, visto que o autor foi responsável por quebrar paradigmas econômicos criados por Adam Smith, ao criar a “ Teoria do Equilíbrio de Nash" que propões a possibilidade de ações colaborativas entre os agentes em jogos nãocooperativos, o que expandiu o escopo da Teoria do Jogos, uma vez que a noção de equilíbrio defendida pelo autor não se restringia apenas aos jogos de soma zero.

O equilíbrio de Nash é verificado quando a combinação de estratégias escolhidas pelo jogador é aquela na qual nenhum jogador faria melhor escolhendo uma alternativa diferente dada a estratégia que o outro escolhe. De modo que, a cooperação entre os jogadores possibilita a maximização de interesses de ambos.

Demostrou ser viável a aplicação de conceitos da teoria dos jogos ao instituto da mediação. Nesse sentido, verificou-se que a teoria dos jogos pode ser utilizada para compreender a função da mediação, bem como para ser mais uma ferramenta voltada para cooperação das partes, com o intuito de possibilitar a maximização da utilidade de todos os envolvidos.

Vale ressaltar que, mesmo nos casos em que, aplicando-se a teoria dos jogos, a mediação não coloque um fim no litígio, esta se mostra eficiente, visto que auxilia na retomada da comunicação, reduzindo a assimetria informacional, e assim, reduzindo a contenda.

Por fim, conclui-se que a a opção pela cooperação, proposto pela teoria de Nash em sendo aplicada ao instituto da mediação, é mais racional e economicamente eficiente. Uma vez que possui como consequência a otimização de resultados, gerando uma solução de ganhos mútuos, mais eficiente com maximização dos interesses individuais para todos os envolvidos, os quais, se agissem de forma não cooperativa, acabariam obtendo resultados menos eficientes. 


\section{REFERÊNCIAS}

ALMEIDA, Fábio Portela Lopes de. A teoria dos jogos: uma fundamentação teórica dos métodos de resolução de disputa. Disponível em: http:// www.arcos.org.br/livros/estudosdearbitragem- mediacao-enegociacao-vol2/terceira-parte-artigo-dos-pesquisadores/ateoriadosjogos-uma-fundamentacao-teoricados-metodos-de-resolucaode-disputa. Acesso em: ago 2021.

AYRES, Ian; BROWN, Jennifer Gerarda. Economic rationales for mediation. (1994). Yale Law Faculty Scholarship Se-ries. Paper 1529.

AZEVEDO, 2015 apud. BORBA Janine Taís Homem Echevarria; FIBRANS, William Picolo; COSTA Dnda. Thaíse Nara Graziottin. A Mediação De Conflitos: Um Olhar A Partir Da Teoria Dos Jogos. Mostra de Inovação Cientifica -IMED, 2016.

BAIRD, Douglas; GERTNER, Robert H.; e PICKER, Randal C. Game theory and the law. Cambridge, MA: Harvard Uni-versity Press, 1994.

BACELLAR, Roberto Portugal. Col. saberes do direito 53 - Mediação e Arbitragem, 1ed. São Paulo: Saraiva, 2012.

BORBA Janine Taís Homem Echevarria; FIBRANS, William Picolo; COSTA Dnda. Thaíse Nara Graziottin. A Mediação De Conflitos: Um Olhar A Partir Da Teoria Dos Jogos. Mostra de Inovação Cientifica -IMED, 2016.

BRASIL. Lei n.o 13.140, de 26 de junho de 2015. Dispõe sobre a mediação entre particulares como meio de solução de controvérsias e sobre a autocomposição de conflitos no âmbito da administração pública; altera a Lei no 9.469 , de 10 de julho de 1997, e o Decreto no 70.235, de 6 de março de 1972; e revoga o $\S 2$ o do art. 6o da Lei no 9.469, de 10 de julho de 1997. Disponível em http:// www.planalto.gov.br/cci-vil_03/_ato2015-2018/2015/Lei/L13140.htm Acesso em: 25 de ago 2021.

CASELlA, Paulo Borba; DE SOUZA, Luciane Moessa. Mediação de conflitos. Novo paradigma de acesso à justiça. Belo Horizonte: Editora Fórum, 2009.

CONSELHO NACIONAL DE JUSTIÇA. Manual de Mediação Judicial. 5. ed. Brasília: CNJ, 2015.

DEUTSCH, Morton. The resolution of conflict: constructive and destructive processes. New Haven:Yale University Press, 1973.

DIAS, Henry Paulo. A Teoria dos Jogos. Global Manager Revista do Curso de Administração da

Faculdade da Serra Gaúcha f. 49-56, ano 4, no 6, jun. 2004. 
DÓRIA, Nilma Raidete Souto. A Teoria dos Jogos de Nash Utilizada Como Método Alternativo De Resolução De Conflitos. REVISTA DA ESCOLA DA MAGISTRATURA DO ESTADO DE RONDÔNIA - ANO 2019 - No 26, 2019.

FIANI, Ronaldo. Teoria dos Jogos: com aplicações em Economia, Administração e Serviços Sociais. 4 ed. Rio de Janeiro: Elsevier, 2015.

FERIOLI LAGRASTA LUCHIARI, Valeria. Mediação Judicial. Analise da realidade brasileira. Origem e evolução até a Resolução no 125 do Conselho Nacional de Justiça. Coordenadores Ada Pellegrini Grinover, Kazuo Watanabe. Rio de Janeiro: Editora Forense, 2012.

FISHER, Roger; URY, William, PATTON, Bruce. Como chegar ao sim, como negociar acordos e fazer concessões. Rio de Janeiro; Sextante, 2018.

GUILHERME, Luiz Fernando do Vale de Almeida. Manual de arbitragem e mediação: conciliação e negociação. 5. ed. - São Paulo: Saraiva Educação, 2020.

KESSLER, Daniela Seadi; TRINDATE, Manoel Gustavo Neubarth . A MEDIAÇÃO SOB O PRISMA DA ANÁLISE ECONÔMICA DO DIREITO. RJLB. Ano 5 (2019), no 4, 535-591.

OLIVEIRA, Angela. Métodos de resolução de controvérsias. São Paulo: LTR, 1999.

SALLES, Carlos Alberto de, et. al. Negociação, mediação, conciliação e arbitragem: curso de métodos adequados de solução de controvérsias. 3. ed. Rio de Janeiro: Forense, 2020.

SANTINI, Brígida Alexandre; GARBUGIO, Gilmar; BORTOLOSSI, Humberto José; SANTOS, Polyana Alves; BARRETO,Larissa Santana. 2010 apud DIAS, Henry Paulo. A Teoria dos Jogos. Global Manager Revista do Curso de Administração da Faculdade da Serra Gaúcha f. 49-56, ano 4, no 6, jun. 2004.

SANTOS, 2004 apud. KESSLER, Daniela Seadi; TRINDATE, Manoel Gustavo Neubarth . A MEDIAÇÃO SOB O PRISMA DA ANÁLISE ECONÔMICA DO DIREITO. RJLB. Ano 5 (2019), no 4, 535-591.

TARTUCE, Fernanda. Mediação nos conflitos civis. 6. ed. Rio de Janeiro: Forense; São Paulo: MÉTODO, 2021.

VITALE, Carla Maria Franco Lameir; SILVA, Luciana Aboim Machado Gonçalves. Aplicação da Teoria dos Jogos na Mediação de Conflitos: o Equilíbrio de Nash como Estratégia de Maximização de Ganhos. Revista FONAMEC - Rio de Janeiro, v.1, n. 1, p. 94 - 110, maio 2017 